PROCEEDINGS OF THE

AMERICAN MATHEMATICAL SOCIETY

Volume 128, Number 9, Pages 2667-2673

S 0002-9939(00)05520-9

Article electronically published on March 1, 2000

\title{
ON SOME PROPERTIES OF THE GAMMA FUNCTION
}

\author{
ÁRPÁD ELBERT AND ANDREA LAFORGIA
}

(Communicated by Hal L. Smith)

\begin{abstract}
Anderson and Qiu (1997) conjectured that the function $\frac{\log \Gamma(x+1)}{x \log x}$ is concave for $x>1$. In this paper we prove this conjecture. We also study the monotonicity of some functions connected with the psi-function $\psi(x)$ and derive inequalities for $\psi(x)$ and $\psi^{\prime}(x)$.
\end{abstract}

\section{INTRODUCTION}

For $x>0$ let $\Gamma(x)$ and $\psi(x)$ denote the Euler's gamma function, defined by

$$
\Gamma(x)=\int_{0}^{\infty} e^{-t} t^{x-1} d t, \quad \psi(x)=\frac{\Gamma^{\prime}(x)}{\Gamma(x)},
$$

respectively. There is a vast literature on these functions and a good reference to this can be found, for example, in the recent paper 2 .

Anderson and Qiu showed that the function $\frac{\log \Gamma(x+1)}{x \log x}$ strictly increases from $1-\gamma$ to 1 as $x$ increases from 1 to $\infty$, where $\gamma=0.577 \ldots$ denotes the Euler-Mascheroni constant. To do this, they investigated the function

$$
f(x)=\psi^{\prime}(1+x)+x \psi^{\prime \prime}(1+x)
$$

and they found the representation

$$
f(x)=\sum_{n=1}^{\infty} \frac{n-x}{(n+x)^{3}} .
$$

They proved, in a complicated way, that $f(x)>0$ for $x \in[1,4)$ and formulated the following:

Conjecture. The function $\frac{\log \Gamma(x+1)}{x \log x}$ is concave for $x>1$.

In Section 2 we extend the inequality $f(x)>0$ from $[1,4)$ to $(-1, \infty)$ (this extension is evident for $-1<x \leq 1)$. Then we derive also new inequalities for $\psi(x)$ and $\psi^{\prime}(x)$.

In Section 3 we prove the conjecture formulated above by Anderson and Qiu [3].

Received by the editors October 23, 1998.

2000 Mathematics Subject Classification. Primary 33B15; Secondary 26A48, 26D07.

Key words and phrases. Gamma function, psi function, monotonicity, inequalities.

(C)2000 American Mathematical Society 
We recall now the following two asymptotic representations [1, p. 260; 6.4.12, 6.4.13]:

$$
\begin{gathered}
\psi^{\prime}(z) \sim \frac{1}{z}+\frac{1}{2 z^{2}}+\frac{1}{6 z^{3}}-\frac{1}{30 z^{5}}+\cdots \quad(z \rightarrow \infty,|\arg z|<\pi), \\
\psi^{\prime \prime}(z) \sim-\frac{1}{z^{2}}-\frac{1}{z^{3}}-\frac{1}{2 z^{4}}+\frac{1}{6 z^{6}}-\cdots \quad(z \rightarrow \infty,|\arg z|<\pi),
\end{gathered}
$$

which will be used only for real $z$ 's in the next sections.

\section{NEW RESULTS}

Our first result provides an extension of one proved by Anderson and Qiu [3]. Indeed, we prove, in a simple way, that $f(x)$ is positive not only for $x \geq 1$, but even for $x>-1$.

Theorem 1. Let $f(x)$ be defined by (1.1). Then $f(x)>0$ for any $x>-1$.

Proof. By the asymptotic formulas (1.3) and (1.4), we get

$$
\lim _{x \rightarrow+\infty} f(x)=0 .
$$

So, in order to show that $f(x)>0$, it is sufficient to show that $f(x)-f(x+1)>0$. By (1.2) we get

$$
f(x)-f(x+1)=-\frac{1}{(1+x)^{2}}+\sum_{n=1}^{\infty} \frac{2}{(n+x)^{3}} .
$$

Hence, by the inequality

$$
\frac{2}{u^{3}}>\frac{1}{u^{2}}-\frac{1}{(1+u)^{2}}, \quad u>0,
$$

we find that

$$
f(x)-f(x+1)>-\frac{1}{(1+x)^{2}}+\sum_{n=1}^{\infty}\left[\frac{1}{(n+x)^{2}}-\frac{1}{(n+x+1)^{2}}\right]=0 .
$$

The proof is complete.

Now we use this result to derive the following

Theorem 2. Let the function $g(x)$ be defined by

$$
g(x)=x^{2} \psi^{\prime}(1+x)-x \psi(1+x)+\log \Gamma(x+1), \quad x>-1 .
$$

Then $g(x)$ strictly decreases from $\infty$ to 0 on $(-1,0]$ and strictly increases from 0 to $\infty$ on $[0, \infty)$.

Proof. By differentiation we get

$$
g^{\prime}(x)=x \psi^{\prime}(1+x)+x^{2} \psi^{\prime \prime}(1+x)=x f(x),
$$

where $f(x)$ is defined in (1.1). 
By Theorem 1, $f(x)>0$ for $x>-1$; hence $g(x)$ is decreasing for $-1<x \leq 0$ and is increasing for $x \geq 0$. Clearly $g(0)=0$, and by [1, 6.3.16] we get

$$
\begin{aligned}
g(x) & =x^{2} \psi^{\prime}(1+x)-x \psi(1+x)+\log \Gamma(1+x) \\
& =x^{2} \sum_{n=1}^{\infty} \frac{1}{(n+x)^{2}}-x\left[-\gamma+\sum_{n=1}^{\infty} \frac{x}{n(n+x)}\right]+\log \Gamma(1+x) \\
& =\sum_{n=1}^{\infty} \frac{-x^{3}}{n(n+x)^{2}}-\gamma x+\log \Gamma(1+x),
\end{aligned}
$$

which tends to $\infty$ as $x$ tends to $-1^{+}$.

By [1, 6.3.5] and [1, 6.1.15] we get

$$
g(x)-\frac{1}{2} \log x=\left[x^{2} \psi^{\prime}(x)-x\right]+x[\log x-\psi(x)]+\log \left(\Gamma(x) e^{x} x^{-x+1 / 2}\right) .
$$

By [1, 6.4.12] the function in the first bracket tends to $1 / 2$, and by Stirling's Formula [1, 6.1.37] the function in the third bracket tends to $\sqrt{2 \pi}$, as $x$ tends to $\infty$. Finally by [2. (2.1)], the middle term tends to $1 / 2$ as $x$ tends to $\infty$. So $g(x)-\frac{1}{2} \log x=\mathrm{O}(1)$ as $x$ tends to $\infty$.

This completes the proof of Theorem 2 .

Theorem 3. Let

$$
h(x)=x^{2} \psi^{\prime}(x+1)+x^{3} \psi^{\prime \prime}(x+1)
$$

for $x>0$. Then

$$
0<h(x)<\frac{1}{2} .
$$

Proof. The lower bound follows by Theorem 1 since $h(x)=x^{2} f(x)$. To prove the upper bound we define the function $r(x)$ by

$$
r(x)=\frac{1}{2 x^{2}}-f(x), \quad x>0 .
$$

Clearly $r(x) \rightarrow 0$ as $x \rightarrow \infty$. Thus it is sufficient to show that $r(x)>r(x+1)$. Making use of the inequality

we obtain

$$
\frac{2}{u^{3}}<\frac{1}{2(u-1)^{2}}-\frac{1}{2(u+1)^{2}}, \quad u>1,
$$

$$
\begin{aligned}
r(x)-r(x+1) & =\frac{1}{2 x^{2}}+\frac{1}{2(1+x)^{2}}-\sum_{n=1}^{\infty} \frac{2}{(n+x)^{3}} \\
& >\frac{1}{2 x^{2}}+\frac{1}{2(1+x)^{2}}-\sum_{n=1}^{\infty}\left[\frac{1}{2(n-1+x)^{2}}-\frac{1}{2(n+1+x)^{2}}\right] \\
& =\frac{1}{2 x^{2}}+\frac{1}{2(1+x)^{2}}-\left[\frac{1}{2 x^{2}}+\frac{1}{2(1+x)^{2}}\right]=0 .
\end{aligned}
$$

The proof of Theorem 3 is complete.

Remark. The inequality $h(x)<\frac{1}{2}$, proved in Theorem 3 , is equivalent to

$$
\left(x \psi^{\prime}(1+x)+\frac{1}{2 x}\right)^{\prime}<0
$$


or to

$$
\left(x \psi^{\prime}(x)-\frac{1}{2 x}\right)^{\prime}<0
$$

Hence

$$
x \psi^{\prime}(x)-\frac{1}{2 x}>\lim _{x \rightarrow \infty}\left(x \psi^{\prime}(x)-\frac{1}{2 x}\right)=1
$$

which leads to the inequality

$$
\psi^{\prime}(x)-\frac{1}{x}-\frac{1}{2 x^{2}}>0
$$

This inequality is the same that we obtain using the fact that the second derivative of the function

$$
S_{0}(x)=\log \Gamma(x)-\left(x-\frac{1}{2}\right) \log x+x-\frac{1}{2} \log (2 \pi)
$$

is positive, a result which follows from the complete monotonicity property of $S_{0}(x)$ proved by Muldoon [5. Theorem 8].

\section{Proof of the conjecture}

We are going to prove that

$$
\left(\frac{\log \Gamma(x+1)}{x \log x}\right)^{\prime \prime}<0, \quad \text { for } x>1 .
$$

To this end we distinguish two cases:

$$
\begin{aligned}
& \text { a) } 1<x \leq 2 \\
& \text { b) } x>2 \text {. }
\end{aligned}
$$

Proof of (3.1) in case a). We consider the infinite product representation [4, p. 37]

$$
\Gamma(1+x)=\prod_{n=1}^{\infty} \frac{\left(1+\frac{1}{n}\right)^{x}}{1+\frac{x}{n}}, \quad x \neq-1,-2, \cdots .
$$

Taking the logarithm, we find

$$
\frac{\log \Gamma(x+1)}{x \log x}=\sum_{n=1}^{\infty} f\left(x, \frac{1}{n}\right)
$$

where

$$
f(x, t)=\frac{x \log (1+t)-\log (1+t x)}{x \log x}, \quad 0 \leq t \leq 1 .
$$

If for some $x$ we prove that

$$
\frac{\partial^{2}}{\partial x^{2}} f\left(x, \frac{1}{n}\right)<0, \quad \text { for } n=1,2, \cdots,
$$

then the inequality (3.1) holds for that value of $x$. Since $f(x, 0) \equiv 0$, we have $\frac{\partial^{2}}{\partial x^{2}} f(x, 0) \equiv 0$. We are going to show the inequality

$$
\frac{\partial^{2}}{\partial x^{2}} f(x, t)<0, \quad \text { for } 1 \leq x \leq 2,0<t \leq 1 .
$$

To this end we show that $\frac{\partial^{2}}{\partial x^{2}} f(x, t)$ is a decreasing function of $t$, at least for $1 \leq x \leq 2, x$ fixed. 
Indeed, we have

$$
\begin{gathered}
\frac{\partial}{\partial t} \frac{\partial^{2}}{\partial x^{2}} f(x, t)=\frac{\partial^{2}}{\partial x^{2}} \frac{\partial}{\partial t} f(x, t)=\frac{\partial^{2}}{\partial x^{2}} \frac{t x-t}{(1+t)(1+t x) \log x} \\
\quad=\frac{t}{t+1} \frac{D(x, t)}{x^{2}(1+x t)^{3} \log ^{3} x},
\end{gathered}
$$

where

$$
\begin{aligned}
& D(x, t)=a(x)+b(x) t+c(x) t^{2}, \\
& a(x)=-2+2 x-(x+1) \log x, \\
& b(x)=2 x\left(2 x-2-2 \log x-x \log ^{2} x\right), \\
& c(x)=x^{2}\left(2 x-2-3 \log x+x \log x-2 \log ^{2} x\right) .
\end{aligned}
$$

We observe that $a(x)<0$ and $c(x)>0$ for $x>1$. Indeed $a(1)=0$ and $a^{\prime}(x)<0$ and therefore $a(x)<0$, for $x>1$. Concerning $c(x)$ we have $c(1)=0$ and $\left(\frac{c(x)}{x^{2}}\right)^{\prime}>0$. We have to show that $D(x, t)<0$. The function $D(x, t)$ is convex with respect to $t$; therefore we have only to show that $D(x, 0)<0$ and $D(x, 1)<0$.

For $t=0$ we get $D(x, 0)=a(x)<0$. Moreover

$$
\begin{aligned}
d(x) & =D(x, 1)=a(x)+b(x)+c(x) \\
& =2(x+1)^{2}(x-1)+\left(x^{3}-3 x^{2}-5 x-1\right) \log x-4 x^{2} \log ^{2} x
\end{aligned}
$$

and we need to show that $d(x)<0$. It is easy to check that $d(1)=0, d(2)=$ $18-15 \log 2-16 \log ^{2} 2=-0.084 \ldots<0$. By $(3.2)$

$$
\left(\frac{d(x)}{x^{2}}\right)^{\prime}=\frac{x-1}{x^{3}}\left[3 x^{2}-3+\left(x^{2}-7 x-2\right) \log x\right]=\frac{x-1}{x^{3}} d_{1}(x),
$$

where $d_{1}(1)=0, d_{1}(2)=9-12 \log 2=0.682 \ldots>0$, and

$$
\left(\frac{d_{1}(x)}{2+7 x-x^{2}}\right)^{\prime}=-\frac{d_{2}(x)}{x\left(2+7 x-x^{2}\right)^{2}},
$$

where

$$
d_{2}(x)=x^{4}-35 x^{3}+39 x^{2}+7 x+4 .
$$

Since

$$
\begin{aligned}
& d_{2}(1)=16>0, \\
& d_{2}(2)=-90<0, \\
& \lim _{x \rightarrow \infty} d_{2}(x)=+\infty,
\end{aligned}
$$

it follows that $d_{2}(x)$ has a zero in $(1,2)$ and a zero in $(2, \infty)$. By Descartes' Rule of Signs $d_{2}(x)$ can have either no positive zeros or two positive zeros. Thus we conclude that $d_{2}$ has exactly two positive zeros. Consequently $d_{2}(x)$ has only one zero, say $\xi_{2}$, in $(1,2)$. Thus the function $\left(\frac{d_{1}(x)}{2+7 x-x^{2}}\right)^{\prime}$ is negative in $\left(1, \xi_{2}\right)$ and positive in $\left(\xi_{2}, 2\right)$, and in particular $d_{1}\left(\xi_{2}\right)<0$. Since also $d_{1}(2)>0$, we see that $d_{1}(x)$ has exactly one zero $\xi_{1}$ in $(1,2)$. Finally we have that $\left(\frac{d(x)}{x^{2}}\right)^{\prime}$ is negative 
in $\left(1, \xi_{1}\right)$ and positive in $\left(\xi_{1}, 2\right)$. Since also $d(1)=0$ and $d(2)<0$, this shows that $d(x)<0$ in $(1,2)$. This completes the proof of the conjecture for $x \in(1,2]$.

Proof of (3.1) in case b). Direct calculations show that

$$
\left(\frac{\log \Gamma(x+1)}{x \log x}\right)^{\prime \prime}=\frac{2}{x^{3}}+\frac{2+3 l+2 l^{2}}{x^{3} l^{3}} \log \Gamma(x)-2 \frac{l+1}{x^{2} l^{2}} \psi(x)+\frac{1}{x l} \psi^{\prime}(x)
$$

where $l=\log x$, and we have to prove that the right-hand side is negative for $x>2$.

We are looking for an upper bound for $\log \Gamma(x)$, a lower bound for $\psi(x)$ and an upper bound for $\psi^{\prime}(x)$. By [2, p. 383; Theorem 8] we get that the function

$$
G_{0}(x)=-\log \Gamma(x)+\left(x-\frac{1}{2}\right) l-x+\lambda+\frac{1}{12 x},
$$

where

$$
\lambda=\frac{\log (2 \pi)}{2},
$$

is strictly completely monotonic on $(0, \infty)$.

This gives that

$$
\begin{aligned}
& \log \Gamma(x)<\left(x-\frac{1}{2}\right) l-x+\lambda+\frac{1}{12 x}, \\
& \psi(x)>l-\frac{1}{2 x}-\frac{1}{12 x^{2}}, \\
& \psi^{\prime}(x)<\frac{1}{x}+\frac{1}{2 x^{2}}+\frac{1}{6 x^{3}} .
\end{aligned}
$$

Thus replacing $\log \Gamma(x), \psi(x), \psi^{\prime}(x)$ in (3.3) with these bounds, we have

$$
\begin{aligned}
x^{3} l^{3}\left(\frac{\log \Gamma(x+1)}{x \log x}\right)^{\prime \prime} & <2 l^{3}+\left(2+3 l+2 l^{2}\right)\left[\left(x-\frac{1}{2}\right) l-x+\lambda+\frac{1}{12 x}\right] \\
& -2 x l(l+1)\left[l-\frac{1}{2 x}-\frac{1}{12 x^{2}}\right]+x^{2} l^{2}\left(\frac{1}{x}+\frac{1}{2 x^{2}}+\frac{1}{6 x^{3}}\right) \\
& =A(l) x+B(l)+\frac{1}{x} C(l),
\end{aligned}
$$

where

$$
\begin{aligned}
& A(l)=-(l+2), \\
& B(l)=l^{3}+2 \lambda l^{2}+3 \lambda l+2 \lambda, \\
& C(l)=\frac{1}{2} l^{2}+\frac{5}{12} l+\frac{1}{6} .
\end{aligned}
$$

Let us consider the quadratic polynomial

$$
Q(z)=A(l) z^{2}+B(l) z+C(l) .
$$

By (3.4) we have to show that $Q(x)<0$ for $x>2$. Since $A(l)<0$ and $C(l)>0$, the polynomial $Q(z)$ has exactly one positive zero $z_{0}$ and $Q(z)$ is negative when $z>z_{0}$. The Taylor polynomial of the second degree for $e^{l}$ at $l=\log 2$ gives a lower bound for $e^{l}, l>\log 2$. Thus we find

$$
x=e^{l}>l^{2}+\mu l+\nu \equiv z_{1}, \quad x>2,
$$


where

$$
\mu=2-2 \log 2, \quad \nu=1+(1-\log 2)^{2} .
$$

Using the numerical values of $\mu$ and $\nu$ we obtain

$$
Q\left(z_{1}\right)<-0.775828 l^{4}-0.040884 l^{3}-0.432207 l^{2}+0.677828 l-0.216770
$$

and we wish to show that $Q\left(z_{1}\right)<0$ for $l>\log 2$. Using the inequalities

$$
l^{4} \geq l^{2} \log ^{2} 2, \quad l^{3} \geq l^{2} \log 2,
$$

we get

$$
Q\left(z_{1}\right)<-0.833295 l^{2}+0.677828 l-0.216770 .
$$

Since the polynomial on the right-hand side has no real zeros, we have $Q\left(z_{1}\right)<$ 0 . This shows that $z_{1}>z_{0}$ and consequently $Q(x)<0$, because by $(3.5), x>z_{1}$. This completes the proof of the conjecture.

\section{ACKNOWLEDGEMENTS}

The autors thank the referee for the careful reading of the manuscript and for the helpful comments.

\section{REFERENCES}

[1] M. Abramowitz and I. A. Stegun, eds., Handbook of Mathematical Functions, with Formulas, Graphs, and Mathematical Tables, Dover, New York, 1965. MR 31:1400

[2] H. Alzer, On some inequalities for the gamma and psi functions, Math. Comp. 66 (1997), 373-389. MR 97e:33004

[3] G. D. Anderson and S.-L. Qiu, A monotoneity property of the gamma function, Proc. Amer. Math. Soc. 125 (1997), 3355-3362. MR 98h:33001]

[4] B. C. Carlson, Special Functions of Applied Mathematics, Academic Press, New York, 1977. MR 58:28707

[5] M. E. Muldoon, Some monotonicity properties and characterizations of the gamma function, Aequationes Math. 18 (1978), 54-63. MR 58:11536]

Mathematical Institute of the Hungarian Academy of Sciences, P.O. Box 127, BuDAPEST H-1364, HUNGARY

Department of Mathematics, Largo S. Leonardo Murialdo, 100146 Roma, Italy

E-mail address: laforgia@mat.uniroma3.it 